\title{
Positive and Negative Integrable Hierarchies: Bi-Hamiltonian Structure and Darboux-Bäcklund Transformation
}

\author{
Ning Zhang' and Xi-Xiang Xu $\mathbb{D}^{2}$ \\ ${ }^{1}$ Public Course Teaching Department, Shandong University of Science and Technology, Taian 271019, China \\ ${ }^{2}$ College of Mathematics and Systems Science, Shandong University of Science and Technology, Qingdao 266590, China
}

Correspondence should be addressed to Xi-Xiang Xu; xixiang_xu@sohu.com

Received 21 May 2020; Accepted 14 December 2020; Published 30 December 2020

Academic Editor: Irfan Kaymaz

Copyright (c) 2020 Ning Zhang and Xi-Xiang Xu. This is an open access article distributed under the Creative Commons Attribution License, which permits unrestricted use, distribution, and reproduction in any medium, provided the original work is properly cited.

Two integrable hierarchies are derived from a novel discrete matrix spectral problem by discrete zero curvature equations. They correspond, respectively, to positive power and negative power expansions of Lax operators with respect to the spectral parameter. The bi-Hamiltonian structures of obtained hierarchies are established by a pair of Hamiltonian operators through discrete trace identity. The Liouville integrability of the obtained hierarchies is proved. Through a gauge transformation of the Lax pair, a Darboux-Bäcklund transformation is constructed for the first nonlinear different-difference equation in the negative hierarchy. Ultimately, applying the obtained Darboux-Bäcklund transformation, two exact solutions are given by means of mathematical software.

\section{Introduction}

It is well known that the study of nonlinear integrable differential-difference equations (NIDDEs) has attracted much attention in recent decades [1-14]. Many problems in mathematical physics may be modeled by NIDDEs. Up to now, many important NIDDEs have been presented such as the Ablowitz-Ladik lattice [1], the Toda lattice [2], the relativistic Toda lattice [3], the modified Toda lattice [4, 5], the Merola-Ragnisco-Tu lattice [6], and the deformed reduced semidiscrete Kaup-Newell lattice [7-14]. Now, finding new NIDDEs, in lattice soliton theory, is still an important and complicated task. In general, we choose an appropriate discrete matrix spectral problem and a list of auxiliary spectral problems:

$$
\begin{aligned}
& E \varphi_{n}=\varphi_{n+1}=U_{n}\left(u_{n}, \lambda\right) \varphi_{n}, \\
& \varphi_{n_{t_{m}}}=V_{n}^{(m)}\left(u_{n}, \lambda\right) \varphi_{n},
\end{aligned}
$$

where for a lattice function $f_{n}=f(n)$, the shift operator $E$ and the inverse of $E$ are defined by

$$
E f_{n}=f_{n+1}, E^{-1} f_{n}=f_{n-1}, \quad n \in Z,
$$

$U_{n}\left(u_{n}, \lambda\right)$ is a square matrix, $V_{n}^{(m)}\left(u_{n}, \lambda\right)$ is a list of the same order square matrices of $U_{n}\left(u_{n}, \lambda\right), u_{n}$ is a potential vector function, $\varphi_{n}$ is the eigenfunction vector, $\lambda$ is the spectral parameter, and $\lambda_{t}=0$. The integrability condition of (1) is $E\left(\left(\varphi_{n}\right)_{t_{m}}\right)=\left(E \varphi_{n}\right)_{t_{m}}$, and it is equivalent to

$$
U_{n_{t_{m}}}=\left(E V_{n}^{(m)}\right) U_{n}-U_{n} V_{n}^{(m)}, \quad m \geq 0
$$

Here, (3) is called a discrete zero curvature equation. Usually, (3) determines a hierarchy of NIDDEs (or lattice soliton equations):

$$
u_{n_{t_{m}}}=K\left(u_{n}, u_{n-1}, u_{n+1}, \ldots\right)
$$

One of the important problems in the lattice soliton theory is to search for a Hamiltonian operator $J_{1}$ and a hierarchy of conserved densities $\left\{\tilde{H}_{m}^{(m)}\right\}_{m=0}^{\infty}$ so that (4) has the following Hamiltonian structures:

$$
u_{n_{t_{m}}}=J_{1} \frac{\delta \widetilde{H}_{n}^{(m)}}{\delta u_{n}}, \quad m \geq 0
$$


where the Hamiltonian functionals $\tilde{H}_{n}^{(m)}=\sum_{n \in Z} H_{n}^{(m)}$ $(m \geq 1)$ and the variational derivative $\left(\delta \tilde{H}_{n}^{(m)} / \delta u_{n}\right)^{2}=$ $\sum_{m \in Z} E^{-m}\left(\partial H_{n}^{(m)} / \partial u_{n+m}\right)$. Furthermore, if there is another operator $J_{2}$ so that $J_{1}$ and $J_{2}$ form a pair of Hamiltonian operators and

$$
u_{n_{t_{m}}}=J_{1} \frac{\delta \widetilde{H}_{n}^{(m)}}{\delta u_{n}}=J_{2} \frac{\delta \widetilde{H}_{n}^{(m-1)}}{\delta u_{n}}, \quad m \geq 1,
$$

then the integrable hierarchy (4) possess a bi-Hamiltonian structure. According to the theory of Hamiltonian operators, if $J_{1}$ is reversible, then the hierarchy (4) is integrable in Liouville sense $([4,13]$ and their references). As is known to all, staring from a continuous matrix spectral problem, we only derive one integrable hierarchy, but for some suitable discrete matrix spectral problems, we can get two hierarchies of the NIDDEs [13]. In this paper, we are going to present two integrable hierarchies from a discrete matrix spectral problem. They, respectively, apply positive power and negative power expansions of Lax operators with respect to the spectral parameter. Theory is, respectively, called positive and negative integrable hierarchies. Moreover, as is known to all, Bäcklund transformation is a powerful method to obtain exact solutions of NIDDEs $[15,16]$. This transformation is a relation between the new solution and the old solution of the NIDDEs. Based on a known solution, applying this transformation, a new solution may be derived. According to [15], Bäcklund transformation is usually divided into three types: the Wahlquist-Estabrook (WE) type $[16,17]$, the Hirota type [18], and the Darboux-Bäcklund type [19-22]. In the Darboux-Bäcklund type, the Lax pair plays a key role. A gauge transformation of the Lax pairs is called a Darboux-Bäcklund transformation if it transforms the Lax pair into another Lax pair of the same form.

This paper is organized as follows. In Sections 2 and 3, we introduce a novel discrete spectral problem:

$$
\begin{aligned}
E \varphi_{n} & =U_{n}\left(u_{n}, \lambda\right) \varphi_{n}, \\
U_{n}\left(u_{n}, \lambda\right) & =\left(\begin{array}{ll}
p_{n} \lambda & 1 \\
q_{n} \lambda & 1
\end{array}\right),
\end{aligned}
$$

where $\varphi_{n}=\left(\varphi_{n}^{1}, \varphi_{n}^{2}\right)$ is the eigenfunction vector, $u_{n}=\left(p_{n}, q_{n}\right)^{T}$ is the potential vector, and $p_{n}=p(n, t)$ and $q_{n}=q(n, t)$ depend on integer $n \in Z$ and real $t \in R$. Staring from spectral problem (7), positive and negative integrable hierarchies of NIDDEs are, respectively, presented by discrete zero curvature equations. Then, the Hamiltonian structure and bi-Hamiltonian structure of the obtained hierarchies are established by means of the discrete trace identity [11]. Afterwards, infinitely many common commuting conserved functionals of the obtained positive hierarchy are worked out. The Liouville integrability of the obtained positive hierarchy is proved. For the obtained negative integrable hierarchy, the same results can be similarly obtained. In Section 4, a Darboux-Bäcklund transformation is established though the gauge transformation of the Lax pair for the first NIDDE in the negative integrable hierarchy. In Section 5, using obtained Darboux-Bäcklund transformation, two exact solutions are given with the help of the mathematical software "Mathematica." Finally, in Section 6, there will be some conclusions and remarks.

\section{Positive Integrable Hierarchy and Its Bi- Hamiltonian Structure}

Now, we want to deduce a hierarchy of NIDDEs associated with eigenvalue problem (7). For this purpose, we first solve the following stationary discrete zero curvature equation:

$$
\left(E\left(M_{n}\right)\right) U_{n}-U_{n} M_{n}=M_{n+1} U_{n}-U_{n} M_{n}=0 .
$$

Let us set

$$
M_{n}=\left(\begin{array}{cc}
A_{n} & B_{n} \\
C_{n} & -A_{n}
\end{array}\right) .
$$

We find that equation (8) implies

$$
\begin{gathered}
p_{n}\left(A_{n+1}-A_{n}\right)-C_{n}+q_{n} B_{n+1}=0, \\
p_{n} B_{n} \lambda-\left(A_{n+1}+A_{n}\right)-B_{n+1}=0, \\
p_{n} C_{n+1} \lambda-q_{n}\left(A_{n+1}+A_{n}\right)-C_{n}=0, \\
\left(C_{n+1}-q_{n} B_{n}\right) \lambda+\left(A_{n}-A_{n+1}\right)=0 .
\end{gathered}
$$

Substituting expansions

$$
\begin{aligned}
& A_{n}=\sum_{m=0}^{\infty} A_{n}^{(m)} \lambda^{-m}, \\
& B_{n}=\sum_{m=0}^{\infty} B_{n}^{(m)} \lambda^{-m}, \\
& C_{n}=\sum_{m=0}^{\infty} C_{n}^{(m)} \lambda^{-m},
\end{aligned}
$$

into (10) and comparing each power of $\lambda$ in equation (10), we obtain the initial conditions:

$$
p_{n}\left(A_{n+1}^{(0)}-A_{n}^{(0)}\right)=C_{n}^{(0)}-q_{n} B_{n+1}^{(0)}, \quad B_{n}^{(0)}=0, C_{n+1}^{(0)}=0,
$$

and the recursion relations:

$$
\begin{aligned}
p_{n}\left(A_{n+1}^{(m+1)}-A_{n}^{(m+1)}\right) & =C_{n}^{(m+1)}-q_{n} B_{n+1}^{(m+1)}, \quad m \geq 0 \\
p_{n} B_{n}^{(m+1)} & =\left(A_{n}^{(m)}+A_{n+1}^{(m)}\right)+B_{n+1}^{(m)}, \quad m \geq 0, \\
p_{n} C_{n+1}^{(m+1)} & =q_{n}\left(A_{n}^{(m)}+A_{n+1}^{(m)}\right)+C_{n}^{(m)}, \quad m \geq 0 .
\end{aligned}
$$

Proposition 1. We take that

$$
\begin{aligned}
& A_{n}^{(0)}=\frac{1}{2}, \\
& C_{n}^{(0)}=0,
\end{aligned}
$$

then $A_{n}^{(m)}, B_{n}^{(m)}, C_{n}^{(m)}(m \geq 0)$, which are solved by equation (13), are all local, and they are just rational functions in the two dependent variables $p_{n}$ and $q_{n}$. 
Proof. According to second and third equations in (13), we get that $B_{n}^{(m+1)}$ and $C_{n}^{(m+1)}$ can be shown locally by means of $A_{n}^{(m)}, B_{n}^{(m)}$, and $C_{n}^{(m)}(m \geq 0)$. In order to derived $A_{n}^{(m+1)}(m \geq 0)$ from first equation in (13), we require to apply operator $D^{-1}=(E-1)^{-1}$ to solve the related difference equation. Next, we will show that $A_{n}^{(m+1)}(m \geq 0)$ may be also deduced through an algebraic method rather than by solving the difference equation. Based on (8), we obtain that

$$
(E-1) \operatorname{tr}\left(M_{n}^{2}\right)=2(E-1)\left(A_{n}^{2}+B_{n} C_{n} \lambda\right)=0 .
$$

So $\left(A_{n}^{2}+B_{n} C_{n} \lambda\right)=\gamma(t), \gamma(t)$ is an arbitrary function of time variable $t$ only. Furthermore, we take that $\gamma(t)=0$. Then, we obtain a recursion relation for $A_{n}^{(m)}$ :

$$
A_{n}^{(m+1)}=\sum_{j=1}^{m} A_{n}^{(j)} A_{n}^{(m-j+1)}-\sum_{j=1}^{m+1} B_{n}^{(j)} C_{n}^{(m-j+2)}, \quad m \geq 0 .
$$

Therefore, $A_{n}^{(m+1)}(m \geq 0)$ can be derived locally by $A_{n}^{(m)}, B_{n}^{(m)}, \quad$ and $\quad C_{n}^{(m)}(m \geq 0)$ and then $A_{n}^{(m)}, B_{n}^{(m)}$, and $C_{n}^{(m)}(m \geq 0)$ are all local; they are just rational functions in the two dependent variables $p_{n}$ and $q_{n}$. The proof is completed.

Specially, we have

$$
\begin{aligned}
& A_{n}^{(1)}=\frac{-q_{n-1}}{p_{n-1} p_{n}}, B_{n}^{(1)}=\frac{1}{p_{n}}, C_{n}^{(1)}=\frac{q_{n-1}}{p_{n-1}}, A_{n}^{(2)}=\frac{q_{n-1}^{2}}{p_{n-1}^{2} p_{n}^{2}}+\frac{q_{n-1} q_{n}}{p_{n+1} p_{n}^{2} p_{n-1}}+\frac{q_{n-1} q_{n-2}}{p_{n} p_{n-1}^{2} p_{n-2}}-\frac{q_{n-1}}{p_{n} p_{n+1} p_{n-1}}-\frac{q_{n-2}}{p_{n} p_{n-1} p_{n-2}}, \\
& B_{n}^{(2)}=-\frac{q_{n}}{p_{n+1} p_{n}^{2}}-\frac{q_{n-1}}{p_{n}^{2} p_{n-1}}+\frac{1}{p_{n} p_{n+1}}, C_{n}^{(2)}=-\frac{q_{n-1}^{2}}{p_{n-1}^{2} p_{n}}-\frac{q_{n-1} q_{n-2}}{p_{n-1}^{2} p_{n-2}}+\frac{q_{n-2}}{p_{n-1} p_{n-2}} \cdots
\end{aligned}
$$

Let us denote

$M_{n}^{(m)}=\left(\sum_{i=0}^{m} A_{n}^{(i)} \lambda^{m-} \sum_{i=0}^{m} B_{n}^{(i)} \lambda^{m-i} \sum_{i=0}^{m} C_{n}^{(i)} \lambda^{m-i+1}-\sum_{i=0}^{m} A_{n}^{(i)} \lambda^{m-i}\right), \quad m \geq 0$.

By means of (13), we get

$$
\left(E M_{n}^{(m)}\right) U_{n}-U_{n} M_{n}^{(m)}=\left(\begin{array}{cc}
0 & p_{n} B_{n}^{(m+1)} \\
-p_{n} C_{n+1}^{(m+1)} \lambda & \left(A_{n}^{(m)}-A_{n+1}^{(m)}\right)
\end{array}\right) .
$$

Obviously, (19) is not compatible with $\left(U_{n}\right)_{t_{m}}$. So, we choose a correction term

$$
\Delta_{n}^{(m)}=\left(\begin{array}{cc}
-B_{n}^{(m)}-A_{n}^{(m)} & 0 \\
0 & A_{n}^{(m)}
\end{array}\right),
$$

and set

$$
\widetilde{M}_{n}^{(m)}=M_{n}^{(m)}+\Delta_{n}^{(m)}, \quad m \geq 0 .
$$

We consider the following auxiliary spectral problems associated with the spectral problem (7):

$$
\varphi_{n_{t_{m}}}=\widetilde{M}_{n}^{(m)} \varphi_{n}, \quad m \geq 0 .
$$
(22)

Then, the compatibility condition of equations (7) and

$$
\left(E \varphi_{n}\right)_{t_{m}}=E\left(\left(\varphi_{n}\right) t_{m}\right),
$$

is equivalent to the discrete zero curvature equations

$$
U_{n_{t_{m}}}=\left(E \widetilde{M}_{n}^{(m)}\right) U_{n}-U_{n} \widetilde{M}_{n}^{(m)}, \quad m \geq 0,
$$

which give rise to the hierarchy of NLDDEs:

$$
\left\{\begin{array}{l}
p_{n_{t_{m}}}=p_{n}(1-E)\left(A_{n}^{(m)}+B_{n}^{(m)}\right), \\
q_{n_{t_{m}}}=q_{n} B_{n}^{(m)}-C_{n}^{(m)},
\end{array} \quad m \geq 0 .\right.
$$

Remark 1. Owing to the entries in matrix $\widetilde{M}_{n}^{(m)}(m \geq 0)$ only has nonnegative power powers of the eigenvalue $\lambda$, then the integrable hierarchy (25) is called a positive integrable hierarchy associated with the discrete matrix spectral problem (7).

When $m=0$, (25) becomes a trivial linear system:

$$
\left\{\begin{array}{l}
p_{n_{t_{0}}}=0 \\
q_{n_{t_{0}}}=0 .
\end{array}\right.
$$

When $m=1$ in (25), we obtain the first NIDDE in hierarchy (25):

$$
\left\{\begin{array}{l}
p_{n_{t_{1}}}=\frac{p_{n}\left(p_{n-1}-q_{n-1}\right)}{p_{n-1} p_{n}}-\frac{p_{n}\left(p_{n}-q_{n}\right)}{p_{n} p_{n+1}} \\
q_{n_{t_{1}}}=\frac{q_{n}}{p_{n}}-\frac{q_{n-1}}{p_{n-1}}
\end{array}\right.
$$

Furthermore, it is easy to derive the time part of the Lax pair of (27) is

$$
\varphi_{n_{t_{1}}}=\widetilde{M}_{n}^{(1)} \varphi_{n}=\left(\begin{array}{ll}
\frac{\lambda}{2}-\frac{1}{p_{n}} & \frac{1}{p_{n}} \\
\frac{q_{n-1}}{p_{n-1}} \lambda & -\frac{\lambda}{2}
\end{array}\right) \varphi_{n} .
$$


Next, we are going to establish the Hamiltonian structure for the hierarchy of NLDDE (25) by means of the discrete trace identity [11].

First, let us introduce some notions for further discussion. The Gateaux derivative and the inner product are defined, respectively, by

$$
\begin{aligned}
J^{\prime}\left(u_{n}\right)\left[v_{n}\right] & =\left.\frac{\partial}{\partial \varepsilon} J\left(u_{n}+\varepsilon v_{n}\right)\right|_{\varepsilon=0}, \\
\left\langle f_{n}, g_{n}\right\rangle & =\sum_{n \in Z}\left(f_{n}, g_{n}\right)_{R^{2}},
\end{aligned}
$$

where $f_{n}$ and $g_{n}$ are demanded to be rapidly vanished at the infinity. The standard inner product of $f_{n}$ and $g_{n}$ in the Euclidean space $R^{2}$ is given by $\left(f_{n}, g_{n}\right)_{R^{2}}$. Operator $J^{*}$ is defined by $\left\langle f_{n}, J^{*} g_{n}\right\rangle=\left\langle J f_{n}, g_{n}\right\rangle$, and it is called the adjoint operator of $J$. If an operator $J$ possesses the property $J^{*}=-J$, then $J$ is called to be a skew-symmetric. A linear operator $J$ is called a Hamiltonian operator if $J$ is a skewsymmetric operator and fulfills the Jacobi identity, i.e.,

$$
\begin{aligned}
\left\langle f_{n}, J g_{n}\right\rangle & =-\left\langle J f_{n}, g_{n}\right\rangle, \\
\left\langle J^{\prime}\left(u_{n}\right)\left[J f_{n}\right] g_{n}, h_{n}\right\rangle+\operatorname{Cycle}\left(f_{n}, g_{n}, h_{n}\right) & =0 .
\end{aligned}
$$

For a Hamiltonian operator $J$, we may define a corresponding Poisson bracket [4]:

$$
\left\{f_{n}, g_{n}\right\}_{J}=\left\langle\frac{\delta f_{n}}{\delta u_{n}}, J \frac{\delta g_{n}}{\delta u_{n}}\right\rangle=\sum_{n \in Z}\left\langle\frac{\delta f_{n}}{\delta u_{n}}, J \frac{\delta g_{n}}{\delta u_{n}}\right\rangle_{R^{2}}
$$

According to [11], we write

$$
S_{n}=M_{n}\left(U_{n}\right)^{-1}=\left(\begin{array}{cc}
\frac{A_{n}-q_{n} B_{n} \lambda}{\left(p_{n}-q_{n}\right) \lambda} & -\frac{A_{n}-p_{n} B_{n} \lambda}{\left(p_{n}-q_{n}\right) \lambda} \\
\frac{A_{n}+q_{n} C_{n}}{\left(p_{n}-q_{n}\right)} & -\frac{A_{n}+p_{n} C_{n}}{\lambda p_{n}-q_{n}}
\end{array}\right),
$$

and $\langle Y, Z\rangle=\operatorname{Tr}(Y Z)$, where $Y$ and $Z$ are the same order square matrices. We have

$$
\begin{aligned}
& \frac{\partial U_{n}}{\partial \lambda}=\left(\begin{array}{ll}
p_{n} & 0 \\
q_{n} & 0
\end{array}\right), \\
& \frac{\partial U_{n}}{\partial p_{n}}=\left(\begin{array}{ll}
\lambda & 0 \\
0 & 0
\end{array}\right), \\
& \frac{\partial U_{n}}{\partial q_{n}}=\left(\begin{array}{ll}
0 & 0 \\
\lambda & 0
\end{array}\right) .
\end{aligned}
$$

Hence,

$$
\begin{aligned}
& \left\langle R_{n}, \frac{\partial U_{n}}{\partial \lambda}\right\rangle=\frac{A_{n}}{\lambda}, \\
& \left\langle R_{n}, \frac{\partial U_{n}}{\partial p_{n}}\right\rangle=\frac{A_{n}-q_{n} B_{n} \lambda}{p_{n}-q_{n}}, \\
& \left\langle R_{n}, \frac{\partial U_{n}}{\partial q_{n}}\right\rangle=\frac{-A_{n}+p_{n} B_{n} \lambda}{p_{n}-q_{n}} .
\end{aligned}
$$

By virtue of the discrete trace identity [11],

$$
\left\{\begin{array}{c}
\frac{\delta}{\delta p_{n}} \sum_{n \in Z}\left\langle S_{n}, \frac{\partial U_{n}}{\partial \lambda}\right\rangle=\lambda^{-\varepsilon} \frac{\partial}{\partial \lambda} \lambda^{\varepsilon}\left\langle S_{n}, \frac{\partial U_{n}}{\partial p_{n}}\right\rangle \\
\frac{\delta}{\delta q_{n}} \sum_{n \in Z}\left\langle S_{n}, \frac{\partial U_{n}}{\partial \lambda}\right\rangle=\lambda^{-\varepsilon} \frac{\partial}{\partial \lambda} \lambda^{\varepsilon}\left\langle S_{n}, \frac{\partial U_{n}}{\partial q_{n}}\right\rangle
\end{array}\right.
$$

Substituting expansions $A_{n}=\sum_{m=0}^{\infty} A_{n}^{(m)} \lambda^{-m}, B_{n}=$ $\sum_{m=0}^{\infty} B_{n}^{(m)} \lambda^{-m}$, and $C_{n}=\sum_{m=0}^{\infty} C_{n}^{(m)} \lambda^{-m}$ into (35) and comparing the coefficients of $\lambda^{-m-1}$, we arrive at

$$
\left(\begin{array}{c}
\frac{\delta}{\delta p_{n}} \\
\frac{\delta}{\delta q_{n}}
\end{array}\right) \sum_{n \in Z}\left(A_{n}^{(m)}\right)=(\varepsilon-m)\left(\begin{array}{c}
\frac{A_{n}^{(m)}-q_{n} B_{n}^{(m+1)}}{p_{n}-q_{n}} \\
\frac{-A_{n}^{(m)}+p_{n} B_{n}^{(m+1)}}{p_{n}-q_{n}}
\end{array}\right) .
$$

When $m=0$ in equation (36), by means of a direct confirmation, we get that $\varepsilon=0$. Thus, equation (36) can be written as

$$
\left(\begin{array}{c}
\frac{\delta}{\delta p_{n}} \\
\frac{\delta}{\delta q_{n}}
\end{array}\right) \sum_{n \in Z}\left(-\frac{A_{n}^{(m)}}{m}\right)=\left(\begin{array}{c}
\frac{A_{n}^{(m)}-q_{n} B_{n}^{(m+1)}}{p_{n}-q_{n}} \\
\frac{-A_{n}^{(m)}+p_{n} B_{n}^{(m+1)}}{p_{n}-q_{n}}
\end{array}\right), \quad m>0 .
$$

Moreover, we have 


$$
\begin{aligned}
\left(\begin{array}{c}
-p_{n}\left(1-E^{-1}\right)\left(p_{n} B_{n}^{(m+1)}-A_{n}^{(m)}\right) \\
-p_{n} C_{n+1}^{(m+1)}+q_{n} E^{-1}\left(p_{n} B_{n}^{(m+1)}-A_{n}^{(m)}\right)+q_{n} A_{n+1}^{(m)}
\end{array}\right) & =J_{1}\left(\begin{array}{c}
\frac{A_{n}^{(m)}-q_{n} B_{n}^{(m+1)}}{p_{n}-q_{n}} \\
\frac{-A_{n}^{(m)}+p_{n} B_{n}^{(m+1)}}{p_{n}-q_{n}}
\end{array}\right) \\
& =J_{2}\left(\begin{array}{c}
\frac{A_{n}^{(m-1)}-q_{n} B_{n}^{(m)}}{p_{n}-q_{n}}, \\
\frac{-A_{n+1}^{(m-1)}+p_{n} C_{n}^{(m)}}{p_{n}-q_{n}} .
\end{array}\right), m \geq 1,
\end{aligned}
$$

where

$$
\begin{aligned}
& J_{1}=\left(\begin{array}{cc}
0 & -p_{n}^{2}+p_{n} q_{n}+p_{n} E^{-1}\left(p_{n}-q_{n}\right) \\
-p_{n} q_{n}+p_{n}^{2}-\left(p_{n}-q_{n}\right) E p_{n} & -\left(p_{n}-q_{n}\right) E q_{n}+q_{n} E^{-1}\left(p_{n}-q_{n}\right)
\end{array}\right), \\
& J_{2}=\left(\begin{array}{cc}
E^{-1}\left(p_{n}-q_{n}\right)-\left(p_{n}-q_{n}\right) E & p_{n}-q_{n}-\left(p_{n}-q_{n}\right) E \\
-p_{n}+q_{n}+E^{-1}\left(p_{n}-q_{n}\right) & 0
\end{array}\right) .
\end{aligned}
$$

Furthermore, we can get

$$
J_{1}^{-1}=\left(\begin{array}{cc}
Q_{1} & -p_{n}^{2}+p_{n} q_{n}+p_{n} E\left(p_{n}-q_{n}\right) \\
-p_{n} q_{n}+p_{n}^{2}-\left(p_{n}-q_{n}\right) E p_{n} & -\left(p_{n}-q_{n}\right) E q_{n}+q_{n} E^{-1}\left(p_{n}-q_{n}\right)
\end{array}\right),
$$

where $Q_{1}=-\left(1 / p_{n}\right)\left(1-E^{-1}\right)^{-1} E\left(q_{n} /\left(p_{n}-q_{n}\right)\right)(1-E)^{-1}$ $\left(1 / p_{n}\right)-\left(1 / p_{n}\right)\left(1-E^{-1}\right)^{-1}\left(q_{n} /\left(p_{n}-q_{n}\right)\right) E^{-1}(1-E)^{-1}(1 /$ $\left.p_{n}\right)$ and

$$
J_{2}^{-1}=\left(\begin{array}{cc}
0 & -\frac{1}{p_{n}-q_{n}}\left(1-E^{-1}\right)^{-1} \\
(1-E)^{-1} \frac{1}{\left(p_{n}-q_{n}\right)} & Q_{2}
\end{array}\right) .
$$

In above matrix, $Q_{2}=-(1-E)^{-1}\left(1 / \quad\left(p_{n}-q_{n}\right)\right)$ $(1-E)^{-1}+\left(1-E^{-1}\right)^{-1}\left(1 /\left(p_{n}-q_{n}\right)\right)\left(1-E^{-1}\right)^{-1}$.

Proposition 2. For all values of two arbitrary constants $\alpha$ and $\beta$,

$$
J(\alpha, \beta)=\alpha J_{1}+\beta J_{2},
$$

is a Hamiltonian operator.

Expressly, $J_{1}=J(1,0)$ and $J_{2}=J(0,1)$ constitute a pair of Hamiltonian operators.

Proof. Obviously, the operator $J(\alpha, \beta)$ is a skew-symmetric operator, i.e., $J(\alpha, \beta)=-J(\alpha, \beta)^{*}$. Furthermore, by a direct and tedious calculation, we can prove that the operator $J(\alpha, \beta)$ fulfills the Jacobi identity $(30)$.

The proposition is proved.

So we obtain the following proposition.

Proposition 3. Equation (25) possesses the following Hamiltonian structure:

$$
u_{n t_{m}}=\left(\begin{array}{c}
p_{n} \\
q_{n}
\end{array}\right)_{t_{m}}=J_{1}\left(\begin{array}{c}
\frac{A_{n}^{(m)}-q_{n} B_{n}^{(m+1)}}{p_{n}-q_{n}}, \\
\frac{A_{n+1}^{(m)}+C_{n}^{(m+1)}}{p_{n}-q_{n}} .
\end{array}\right)=J_{1} \frac{\delta \tilde{H}_{n}^{(m)}}{\delta u_{n}}, \quad m \geq 1,
$$

where $\widetilde{H}_{n}^{(m)}=\sum_{n \in Z}\left(H_{n}^{(m)}\right)$ and $H_{n}^{(m)}=\left(A_{n}^{(m)} / m\right)(m \geq 1)$.

According to equation (13), we get the recursion relation:

$$
\frac{\delta{\widetilde{H_{n}}}^{(m+1)}}{\delta u_{n}}=\Phi \frac{\delta \widetilde{H}_{n}^{(m)}}{\delta u_{n}}, \quad(m \geq 0), \Phi=J_{1}^{-1} J_{2}
$$

Furthermore, we have 


$$
u_{n, t_{m}}=\left(\begin{array}{c}
p_{n} \\
q_{n}
\end{array}\right)_{t_{m}}=J_{1} \frac{\delta \widetilde{H}_{n}^{(m+1)}}{\delta u_{n}}=J_{2} \frac{\delta \widetilde{H}_{n}^{(m-1)}}{\delta u_{n}}, \quad(m>0) .
$$

That is, (25) is a hierarchy of discrete bi-Hamiltonian systems.

Using the operator $\Phi$, the positive integrable hierarchy (25) can be written as follows:

$$
u_{n_{t_{m}}}=\left(\begin{array}{c}
p_{n} \\
q_{n}
\end{array}\right)_{t_{m}}=J_{1} \frac{\delta \widetilde{H}_{n}^{(m)}}{\delta u_{n}}=J_{1} \Phi \frac{\delta \widetilde{H}_{n}^{(m-1)}}{\delta u_{n}} \cdots=J_{1} \Phi^{m-1} \frac{\delta \widetilde{H}_{n}^{(1)}}{\delta u_{n}}, \quad m>0 .
$$

Obviously, the integrable NLDDE (27) possesses Hamiltonian structure:

$$
u_{n, t_{1}}=\left(\begin{array}{c}
p_{n} \\
q_{n}
\end{array}\right)_{t}=J_{1} \frac{\delta \widetilde{H}_{n}^{(1)}}{\delta u_{n}} .
$$

Next, we prove the Liouville integrability of the discrete bi-Hamiltonian systems (25). It is crucial to make known the existence of infinite involutive conserved functionals.

Proposition 4. $\left\{\widetilde{H}_{n}^{(m)}\right\}_{m=0}^{\infty}$ are conserved functionals of the whole family (25) or (45). And they are in involution in pairs with respect to the Poisson bracket (31).

Proof. Though a direct calculation, we have

$$
\left(J_{1} \Phi\right)^{*}=J_{2}^{*}=-J_{2}=-J_{1} \Phi,
$$

namely,

$$
\Phi^{*} J_{1}=J_{1} \Phi
$$

Therefore,

$$
\begin{aligned}
\left\{\widetilde{H}_{n}^{(m)}, \widetilde{H}_{n}^{(l)}\right\}_{J_{1}} & =\left\langle\frac{\delta \widetilde{H}_{n}^{(m)}}{\delta u_{n}}, J_{1} \frac{\delta \widetilde{H}_{n}^{(l)}}{\delta u_{n}}\right\rangle=\left\langle\Phi^{m-1} \frac{\delta \widetilde{H}_{n}^{(1)}}{\delta u_{n}}, J_{1} \Phi^{l-1} \frac{\delta \widetilde{H}_{n}^{(1)}}{\delta u_{n}}\right\rangle \\
& =\left\langle\Phi^{m-1} \frac{\delta \widetilde{H}_{n}^{(1)}}{\delta u_{n}}, \Phi^{*} J_{1} \Phi^{l-2} \frac{\delta \widetilde{H}_{n}^{(1)}}{\delta u_{n}}\right\rangle=\left\langle\Phi^{m} \frac{\delta \widetilde{H}_{n}^{(1)}}{\delta u_{n}}, J_{1} \Phi^{l-2} \frac{\delta \widetilde{H}_{n}^{(1)}}{\delta u_{n}}\right\rangle \\
& =\left\{\widetilde{H}_{n}^{(m+1)}, \widetilde{H}_{n}^{(l-1)}\right\}_{J_{1}}=\cdots=\left\{\widetilde{H}_{n}^{(m)}, \widetilde{H}_{n}^{(l)}\right\}_{J_{1}}=\left\{\widetilde{H}_{n}^{(m+l-1)}, \widetilde{H}_{n}^{(1)}\right\}_{J_{1}}
\end{aligned}
$$

Repeating the above argument, we can obtain

$$
\left\{\widetilde{H}_{n}^{(l)}, \widetilde{H}_{n}^{(m)}\right\}_{J_{1}}=\left\{\widetilde{H}_{n}^{(m+l-1)}, \widetilde{H}_{n}^{(1)}\right\}_{J_{1}} .
$$

By equations (51) and (52), we have

$$
\begin{gathered}
\left\{\widetilde{H}_{n}^{(m)}, \widetilde{H}_{n}^{(l)}\right\}_{J_{1}}=0, \quad m, l \geq 1, \\
\left(\widetilde{H}_{n}^{(m)}\right)_{t_{l}}=\left\langle\frac{\delta \widetilde{H}_{n}^{(m)}}{\delta u_{n}}, u_{t_{1}}\right\rangle=\left\langle\frac{\delta \widetilde{H}_{n}^{(m)}}{\delta u_{n}}, J_{1} \frac{\delta \widetilde{H}_{n}^{(l)}}{\delta u_{n}}\right\rangle=\left\{\widetilde{H}_{n}^{(m)}, \widetilde{H}_{n}^{(l)}\right\}_{J_{1}}=0, \quad m, l \geq 1 .
\end{gathered}
$$

The proposition is proved.

Based on (45) and the Propositions 3 and 4, we can obtain the following theorem.

Theorem 1. The integrable NLDDE in hierarchy (25) is all Liouville integrable discrete bi-Hamiltonian systems.

\section{Negative Integrable Lattice Hierarchy and Its Bi-Hamiltonian Structure}

In this section, we would like to derive the negative integrable hierarchy associated with matrix spectral problem (7). To this end, we first consider the following stationary discrete zero curvature equation:

$$
\left(E N_{n}\right) U_{n}-U_{n} N_{n}=N_{n+1} U_{n}-U_{n} N_{n}=0,
$$

with

$$
N_{n}=\left(\begin{array}{cc}
a_{n} & b_{n} \\
c_{n} & -a_{n}
\end{array}\right) .
$$

On the basis of equation (54), we arrive at

$$
\begin{aligned}
p_{n}\left(a_{n+1}-a_{n}\right)-c_{n}+q_{n} b_{n+1} & =0, \\
b_{n+1}+\left(a_{n+1}+a_{n}\right)-b_{n} \lambda p_{n} & =0, \\
c_{n}+q_{n}\left(a_{n+1}+a_{n}\right)-p_{n} c_{n+1} \lambda & =0, \\
\left(c_{n+1}-q_{n} b_{n}\right) \lambda+\left(a_{n}-a_{n+1}\right) & =0 .
\end{aligned}
$$


Here, we expand $a_{n}, b_{n}, c_{n}$ by the nonnegative power power of $\lambda$ :

$$
\begin{aligned}
& a_{n}=\sum_{m=0}^{\infty} a_{n}^{(m)} \lambda^{m}, \\
& b_{n}=\sum_{m=0}^{\infty} b_{n}^{(m)} \lambda^{m}, \\
& c_{n}=\sum_{m=0}^{\infty} c_{n}^{(m)} \lambda^{m} .
\end{aligned}
$$

Substituting the above expansions into (56), we get the following initial conditions:

$$
\begin{aligned}
p_{n}\left(a_{n+1}^{(0)}-a_{n}^{(0)}\right)-c_{n}^{(0)}+q_{n} b_{n+1}^{(0)} & =0, \\
b_{n+1}^{(0)} & =-\left(a_{n}^{(0)}+a_{n+1}^{(0)}\right), \\
c_{n} & =-q_{n}\left(a_{n}^{(0)}+a_{n+1}^{(0)}\right),
\end{aligned}
$$

and recursion relations

$$
\begin{aligned}
\left(a_{n+1}^{(m+1)}-a_{n}^{(m+1)}\right) & =c_{n+1}^{(m)}-q_{n} b_{n}^{(m)}, \quad m \geq 0, \\
b_{n+1}^{(m+1)} & =-a_{n+1}^{(m+1)}-a_{n}^{(m+1)}+p_{n} b_{n}^{(m)}, \quad m \geq 0, \\
c_{n}^{(m+1)} & =-q_{n}\left(a_{n}^{(m+1)}+a_{n+1}^{(m+1)}\right)+p_{n} c_{n+1}^{(m)}, \quad m \geq 0 .
\end{aligned}
$$

If the initial values are chosen as

$$
a_{n}^{(0)}=\frac{1}{2}
$$

then we get that

$$
\begin{aligned}
& b_{n}^{(0)}=-1, \\
& c_{n}^{(0)}=-q_{n} .
\end{aligned}
$$

Similar to Proposition 1, we can obtain that $a_{n}^{(m)}, b_{n}^{(m)}, c_{n}^{(m)}(m \geq 1)$ are all local, and they are just polynomial functions in the two dependent variables $p_{n}$ and $q_{n}$, and $\left\{a_{n}^{(m)}\right\}_{m \geq 1}$ may be deduced through an algebraic method rather than by solving the difference equation.

The first few terms are given by

$$
\begin{aligned}
& a_{n}^{(1)}=-q_{n}, \\
& b_{n}^{(1)}=q_{n}+q_{n-1}-p_{n-1}, \\
& c_{n}^{(1)}=q_{n} q_{n+1}+q_{n}^{2}-p_{n} q_{n+1}, \\
& \ldots
\end{aligned}
$$

Set

$$
N_{n}^{(m)}=\left(\sum_{i=0}^{m} a_{n}^{(i)} \lambda^{-m+i-} \sum_{i=0}^{m} b_{n}^{(i)} \lambda^{-m+i-1} \sum_{i=0}^{m} c_{n}^{(i)} \lambda^{-m+i}-\sum_{i=0}^{m} a_{n}^{(i)} \lambda^{-m+i-1}\right), \quad m \geq 0
$$

At this point,

$$
\left(E N_{n}^{(m)}\right) U_{n}-U_{n} N_{n}^{(m)}=\left(\begin{array}{cc}
0 & -p_{n} b_{n}^{(m)} \\
p_{n} c_{n+1}^{(m)} \lambda & c_{n+1}^{(m)}-p_{n} b_{n}^{(m)}
\end{array}\right)
$$

It is obvious that (64) is not also compatible with $\left(U_{n}\right)_{t_{m}}$. So, we choose the following correction term:

$$
\eta_{n}^{(m)}=\left(\begin{array}{cc}
p_{n-1} b_{n-1}^{(m)}-a_{n-1}^{(m+1)} & 0 \\
0 & -a_{n}^{(m+1)}
\end{array}\right) .
$$

Then, we introduce auxiliary matrix spectral problem:

$$
\tilde{N}_{n}^{(m)}=N_{n}^{(m)}+\eta_{n}^{(m)}, \quad m \geq 1 .
$$

Through a direct calculation, we obtain that

$$
\left(U_{n}\right)_{t_{m}}=\left(E \tilde{N}_{n}\right) U_{n}-U_{n} \tilde{N}_{n}, \quad n \geq 0,
$$

it is equivalent to

$$
\left\{\begin{array}{l}
p_{n, t_{m}}=p_{n}\left(1-E^{-1}\right)\left(p_{n} b_{n}^{(m)}-a_{n}^{(m+1)}\right) \\
q_{n, t_{m}}=p_{n} c_{n+1}^{(m)}-q_{n}\left(p_{n-1} b_{n-1}^{(m)}-a_{n-1}^{(m+1)}\right)-q_{n} a_{n+1}^{(m+1)}
\end{array}\right.
$$
$m \geq 0$.

Remark 2. Because the entries in matrix $\tilde{N}_{n}^{(m)}(m \geq 1)$ only have negative powers of the eigenvalue $\lambda$, then the integrable hierarchy (68) is called a negative integrable hierarchy associated with the discrete matrix spectral problem (7).

When $m=0$, (68) becomes

$$
\left\{\begin{array}{l}
p_{n, t_{0}}=p_{n}\left(p_{n-1}-p_{n}\right)-p_{n}\left(q_{n-1}-q_{n}\right) \\
q_{n, t_{0}}=q_{n}\left(q_{n+1}-q_{n-1}\right)+p_{n-1} q_{n}-p_{n} q_{n+1}
\end{array} .\right.
$$

If we set $p_{n}=0,(69)$ is reduced to the well-known Volterra lattice $q_{n_{t}}=q_{n}\left(q_{n+1}-q_{n-1}\right)$; namely, (69) is a generalized Volterra lattice.

In next section, we are going to establish a Darboux-Bäcklund transformation of (69). It is easy to get the time part of the Lax pair of (69) is 


$$
\varphi_{n_{t_{1}}}=\widetilde{N}_{n}^{(1)} \varphi_{n}=\left(\begin{array}{cc}
\frac{1}{2 \lambda}-p_{n-1}+q_{n-1} & -\frac{1}{\lambda} \\
-q_{n} & -\frac{1}{2 \lambda}+q_{n}
\end{array}\right) \varphi_{n} .
$$

In the discrete variational identity (35), we replace $M_{n}$ with $N_{n}$; at this point, the following equations hold:

$$
\left\{\begin{array}{c}
\frac{\delta}{\delta p_{n}} \sum_{n \in Z}\left\langle\widetilde{S}_{n}, \frac{\partial U_{n}}{\partial \lambda}\right\rangle=\lambda^{-\varepsilon} \frac{\partial}{\partial \lambda} \lambda^{\varepsilon}\left\langle\widetilde{S}_{n}, \frac{\partial U_{n}}{\partial p_{n}}\right\rangle, \\
\frac{\delta}{\delta q_{n}} \sum_{n \in Z}\left\langle\widetilde{S}_{n}, \frac{\partial U_{n}}{\partial \lambda}\right\rangle=\lambda^{-\varepsilon} \frac{\partial}{\partial \lambda} \lambda^{\varepsilon}\left\langle\widetilde{S}_{n}, \frac{\partial U_{n}}{\partial q_{n}}\right\rangle,
\end{array}\right.
$$

where $\widetilde{S}_{n}=N_{n}\left(U_{n}\right)^{-1}$.

Substituting expansions

$$
\begin{aligned}
& a_{n}=\sum_{m=0}^{\infty} a_{n}^{(m)} \lambda^{m}, \\
& b_{n}=\sum_{m=0}^{\infty} b_{n}^{(m)} \lambda^{m}, \\
& c_{n}=\sum_{m=0}^{\infty} c_{n}^{(m)} \lambda^{m},
\end{aligned}
$$

into (71), we get

$$
\left(\begin{array}{c}
\frac{\delta \widetilde{K}_{n}^{(m)}}{\delta p_{n}} \\
\frac{\delta \widetilde{K}_{n}^{(m)}}{\delta q_{n}}
\end{array}\right)=\left(\begin{array}{c}
\frac{a_{n}^{(m+1)}-q_{n} b_{n}^{(m)}}{p_{n}-q_{n}} \\
\frac{-a_{n}^{(m+1)}+p_{n} b_{n}^{(m)}}{p_{n}-q_{n}}
\end{array}\right), \quad m \geq 0,
$$

where $\widetilde{K}_{n}^{(m)}=\sum_{n \in Z}\left(-a_{n}^{(m+1)} / m\right)(m \geq 0)$. There is the recursion relation as follows:

$$
\left(\begin{array}{c}
\frac{\delta \widetilde{K}_{n}^{(m)}}{\delta p_{n}} \\
\frac{\delta \widetilde{K}_{n}^{(m)}}{\delta q_{n}}
\end{array}\right)=\Phi^{-1}\left(\begin{array}{c}
\frac{\delta \widetilde{K}_{n}^{(m-1)}}{\delta p_{n}} \\
\frac{\delta \widetilde{K}_{n}^{(m-1)}}{\delta q_{n}}
\end{array}\right), \quad m \geq 0,
$$

where $\Phi^{-1}=J_{2}^{-1} J_{1}$.

Based on (56), we get

$$
\begin{aligned}
\left(\begin{array}{c}
p_{n}\left(1-E^{-1}\right)\left(p_{n} b_{n}^{(m)}-a_{n}^{(m+1)}\right), \\
p_{n} c_{n+1}^{(m)}-q_{n}\left(p_{n-1} b_{n-1}^{(m)} a_{n-1}^{(m+1)}+a_{n+1}^{(m+1)}\right)
\end{array}\right) & =-J_{2}\left(\begin{array}{c}
\frac{a_{n}^{(m+2)}-q_{n} b_{n+1}^{(m+1)}}{p_{n}-q_{n}} \\
\frac{-a_{n}^{(m+2)}+p_{n} b_{n}^{(m+1)}}{p_{n}-q_{n}}
\end{array}\right) \\
& =-J_{1}\left(\begin{array}{c}
\frac{a_{n}^{(m+1)}-q_{n} b_{n+1}^{(m)}}{p_{n}-q_{n}} \\
\frac{-a_{n}^{(m+1)}+p_{n} b_{n+1}^{(m)}}{p_{n}-q_{n}}
\end{array}\right), \quad m \geq 0 .
\end{aligned}
$$

Then, we have

$$
u_{n_{t_{m}}}=\left(\begin{array}{c}
p_{n} \\
q_{n}
\end{array}\right)_{t_{m}}=-J_{2} \frac{\delta \widetilde{K}_{n}^{(m)}}{\delta u_{n}}=-J_{1} \frac{\delta \widetilde{K}_{n}^{(m-1)}}{\delta u_{n}}, \quad m>0 .
$$

Thus, the integrable hierarchy (68) has a bi-Hamiltonian structure (76). Furthermore, similar to integrable hierarchy (25), we can prove that integrable hierarchy (68) is also Liouville integrable.

With the help of the operator $\Phi^{-1}$, the negative integrable hierarchy (68) can be written as follows:

$$
u_{n_{t_{m}}}=\left(\begin{array}{c}
p_{n} \\
q_{n}
\end{array}\right)_{t_{m}}=-J_{2} \frac{\delta \widetilde{K}_{n}^{(m)}}{\delta u_{n}}=-J_{2}\left(\Phi^{-1}\right) \frac{\delta \widetilde{K}_{n}^{(m-1)}}{\delta u_{n}} \cdots=-J_{2}\left(\Phi^{-1}\right)^{m-1} \frac{\delta \widetilde{K}_{n}^{(1)}}{\delta u_{n}}, \quad m>0
$$




\section{Darboux-Bäcklund Transformation}

We introduce a gauge transformation of spectral problem (7):

$$
\widetilde{\varphi}_{n}=T_{n} \varphi_{n}
$$

Under this transformation, two spectral problems (7) and (70) become

$$
\begin{aligned}
E \tilde{\varphi}_{n}=\widetilde{\varphi}_{n+1} & =\widetilde{U}_{n} \widetilde{\varphi}_{n}, \\
\widetilde{\varphi}_{n_{t}} & =\widetilde{N}_{n}^{(1)} \widetilde{\varphi}_{n},
\end{aligned}
$$

with

$$
\begin{aligned}
\widetilde{U}_{n} & =T_{n+1} U_{n}\left(T_{n}\right)^{-1}, \\
\tilde{N}_{n}^{(1)} & =\left(T_{n_{t}}+T_{n} N_{n}^{(1)}\right)\left(T_{n}\right)^{-1} .
\end{aligned}
$$

Here, we suppose

$$
T_{n}=\left(\begin{array}{cc}
\left(T_{n}^{(a)} \lambda+1\right)\left(1-T_{n}^{(b)}\right) & T_{n}^{(b)} \\
T_{n}^{(c)} \lambda & T_{n}^{(d)} \lambda+1
\end{array}\right)
$$

where $T_{n}^{(a)}, T_{n}^{(b)}, T_{n}^{(c)}$, and $T_{n}^{(d)}$ are undetermined functions of variables $n$ and $t$ and $n \in Z, t \in R$. Next, we are going to solve $T_{n}^{(a)}, T_{n}^{(b)}, T_{n}^{(c)}$, and $T_{n}^{(d)}$ such that $\widetilde{U}_{n}$ and $\widetilde{N}_{n}^{(1)}$ in equation (80) are provided with the same form with $U_{n}$ and $N_{n}^{(1)}$, i.e.,

$$
\begin{aligned}
& \widetilde{U}_{n}=\left(\begin{array}{cc}
\tilde{p}_{n} \lambda & 1 \\
\widetilde{q}_{n} \lambda & 1
\end{array}\right), \\
& \tilde{N}^{(1)}=\left(\begin{array}{cc}
\frac{1}{2 \lambda}-\widetilde{p}_{n-1}+\widetilde{q}_{n-1} & -\frac{1}{\lambda} \\
-\widetilde{q}_{n} & -\frac{1}{2 \lambda}+\widetilde{q}_{n}
\end{array}\right) \text {. }
\end{aligned}
$$

For two different reals $\lambda_{1}$ and $\lambda_{2}$, we can get that $y_{n}=$ $\left(y_{n}^{(1)}, y_{n}^{(2)}\right)^{T}$ and $z_{n}=\left(z_{n}^{(1)}, z_{n}^{(2)}\right)^{T}$ are two real linear independent solutions of equations (7) and (70):

$$
\omega_{n}=\left(\begin{array}{cc}
y_{n}^{(1)} & z_{n}^{(1)} \\
y_{2}^{(2)} & z_{n}^{(2)}
\end{array}\right)
$$

Let $\widetilde{\omega}_{n}=T_{n} \omega_{n}$, and we get

$$
\widetilde{\omega}_{n}=\left(\begin{array}{cc}
\left(T_{n}^{(a)} \lambda+1\right)\left(1-T_{n}^{(b)}\right) y_{n}^{(1)}+T_{n}^{(b)} y_{n}^{(2)} & \left(T_{n}^{(a)} \lambda+1\right)\left(1-T_{n}^{(b)}\right) z_{n}^{(1)}+T_{n}^{(b)} z_{n}^{(2)} \\
T_{n}^{(c)} \lambda y_{n}^{(1)}+\left(T_{n}^{(d)} \lambda+1\right) y_{n}^{(2)} & T_{n}^{(c)} \lambda z_{n}^{(1)}+\left(T_{n}^{(d)} \lambda+1\right) z_{n}^{(2)}
\end{array}\right) .
$$

We consider

$$
\begin{array}{cc}
\left.\left(T_{n}^{(a)} \lambda_{i}+1\right)\left(1-T_{n}^{(b)}\right) y_{n}^{(1)}+T_{n}^{(b)} y_{n}^{(2)}=\kappa_{i}\left(T_{n}^{(a)} \lambda_{i}+1\right)\left(1-T_{n}^{(b)}\right) z_{n}^{(1)}+T_{n}^{(b)} z_{n}^{(2)}\right), & \\
T_{n}^{(c)} \lambda y_{n}^{(1)}+\left(T_{n}^{(d)} \lambda_{i}+1\right) y_{n}^{(2)}=\kappa_{i}\left(T_{n}^{(c)} \lambda_{i} z_{n}^{(1)}+\left(T_{n}^{(d)} \lambda_{i}+1\right) z_{n}^{(2)}\right) . & \sigma_{i}[n]=\frac{z_{n}^{(2)}\left(\lambda_{i}\right)-\kappa_{i} y_{n}^{(2)}\left(\lambda_{i}\right)}{z_{n}^{(1)}\left(\lambda_{i}\right)-\kappa_{i} y_{n}^{(1)}\left(\lambda_{i}\right)}, \quad i=1,2 .
\end{array}
$$

In the above equation, set $i=1,2, \kappa_{j}(j=1,2)$, are nonzero constants.

Solving equation (85) for $T_{n}^{(a)}, T_{n}^{(b)}, T_{n}^{(c)}$, and $T_{n}^{(d)}$, we obtain that

$$
\begin{aligned}
& T_{n}^{(a)}=\frac{\sigma_{1}[n]-\sigma_{2}[n]}{\lambda_{1} \sigma_{2}[n]-\lambda_{2} \sigma_{1}[n]} \\
& T_{n}^{(b)}=-\frac{\lambda_{1}-\lambda_{2}}{\lambda_{1}-\lambda_{2}+\left(\lambda_{2} \sigma_{1}[n]-\lambda_{1} \sigma_{2}[n]\right)} \\
& T_{n}^{(c)}=-\frac{\sigma_{1}[n] \sigma_{2}[n]\left(\lambda_{1}-\lambda_{2}\right)}{\lambda_{1} \lambda_{2}\left(\sigma_{1}[n]-\sigma_{2}[n]\right)} \\
& T_{n}^{(d)}=\frac{\lambda_{1} \sigma_{2}[n]-\lambda_{2} \sigma_{1}[n]}{\lambda_{1} \lambda_{2}\left(\sigma_{1}[n]-\sigma_{2}[n]\right)} .
\end{aligned}
$$

In equation (86),
Through direct calculation, we have

$$
\operatorname{Det}\left[T_{n}\right]=\left(1-T_{n}^{(b)}\right) \frac{\left(\lambda-\lambda_{1}\right)\left(\lambda-\lambda_{2}\right)}{\lambda 1 \lambda_{2}} .
$$

Proposition 5. The matrix $\widetilde{U}_{n}$ defined by (80) has the same form as $U_{n}$ in equation (7), and the original potentials $p_{n}$ and $q_{n}$ are changed into new potentials $\widetilde{p}_{n}$ and $\widetilde{q}_{n}$ by means of

$$
\left\{\begin{array}{l}
\widetilde{p}_{n}=-\frac{T_{n}^{(d)} p_{n}}{p_{n} T_{n}^{(b)}+T_{n}^{(a)} T_{n}^{(b)}-T_{n}^{(a)}}, \\
\tilde{q}_{n}=\frac{q_{n}-T_{n}^{(c)}}{1-T_{n}^{(b)}} .
\end{array}\right.
$$


Proof. We know that $T_{n}^{*}$ is the adjoint matrix of $T_{n}$; from equations (7) and (87), we obtain that

$$
\sigma_{i}[n+1]=\frac{\zeta_{i}(n)}{\tau_{i}(n)}, \quad i=1,2,
$$

$\zeta_{i}(n)=\lambda_{i} q_{n}+\sigma_{i}[n], \tau_{i}(n)=\lambda_{i} p_{n}+\sigma_{i}[n], \quad i=1,2$.

From equations (90) and (91), we arrive at

with

$$
\begin{aligned}
T_{n+1}^{(a)} & =\frac{\zeta_{1}(n) \tau_{2}(n)-\zeta_{2}(n) \tau_{1}(n)}{\lambda_{1} \zeta_{2}(n) \tau_{1}(n)-\lambda_{2} \zeta_{1}(n) \tau_{2}(n)} \\
T_{n+1}^{(b)} & =\frac{\left(\lambda_{1}-\lambda_{2}\right) \tau_{1}(n) \tau_{2}(n)}{\left(\lambda_{1}-\lambda_{2}\right) \tau_{1}(n) \tau_{1}(n) \tau_{2}(n)+\lambda_{1} \zeta_{2}(n) \tau_{1}(n)-\lambda_{2} \zeta_{1}(n) \tau_{2}(n)} \\
T_{n+1}^{(c)} & =\frac{\left(\lambda_{1}-\lambda_{2}\right) \zeta_{1}(n) \zeta_{2}(n)}{\lambda_{1} \lambda_{2}\left(\zeta_{1}(n) \tau_{2}(n)-\zeta_{2}(n) \tau_{1}(n)\right.} \\
T_{n+1}^{(d)} & =\frac{\lambda_{1} \lambda_{2}\left(\lambda_{1} \zeta_{2} \tau_{1}(n)-\lambda_{2} \zeta_{1}(n) \tau_{2}(n)\right)}{\lambda_{1} \lambda_{1} \lambda_{2}\left(\zeta_{1}(n) \tau_{2}(n)-\zeta_{2}(n) \tau_{1}(n)\right)}
\end{aligned}
$$

As a result, we have

where

$$
T_{n+1} U_{n} T_{n}^{*}=\left(\begin{array}{ll}
f_{11}(\lambda, n) & f_{12}(\lambda, n) \\
f_{21}(\lambda, n) & f_{22}(\lambda, n)
\end{array}\right),
$$

$$
\begin{aligned}
f_{11}(\lambda, n)= & T_{n+1}^{(a)}\left(1-T_{n+1}^{(b)}\right) T_{n}^{(d)} p_{n} \lambda^{3}+\left(-T_{n+1}^{(a)}\left(1-T_{n+1}^{(b)}\right) T_{n}^{(c)}+T_{n+1}^{(a)}\left(1-T_{n+1}^{(b)}\right) p_{n}+\right. \\
& \left.\left.+\left(1-T_{n+1}^{(b)}\right) T_{n}^{(d)} p_{n}+T_{n+1}^{(b)} D_{n} q_{n}\right) \lambda^{2}+(-) T_{n}^{(c)}+\left(1-T_{n+1}^{(b)}\right) p_{n}+q_{n} T_{n+1}^{(b)}\right) \lambda, \\
f_{12}(\lambda, n)= & T_{n+1}^{(a)}\left(T_{n+1}^{(b)}-1\right)\left(T_{n}^{(a)}\left(\left(T_{n}^{(b)}-1\right)+p_{n} T_{n}^{(b)}\right) \lambda^{2}+\left(T_{n}^{(a)}\right)\left(1-T_{n}^{(b)}\right)-T_{n+1}^{(a)}\left(1-T_{n}^{(b)}\right)\left(T_{n+1}^{(b)}-1\right)\right. \\
& \left.+T_{n}^{(b)}\left(T_{n+1}^{(b)}-1\right) p_{n}-T_{n+1}^{(b)} q_{n}\right) \lambda+1-T_{n}^{(b)}, \\
f_{21}(\lambda, n)= & \left(p_{n} T_{n}^{(d)} T_{n+1}^{(c)}+q_{n} T_{n}^{(d)} T_{n+1}^{(d)}\right) \lambda^{3}+\left(T_{n}^{(c)} T_{n+1}^{(d)}-T_{n+1}^{(c)} T_{n}^{(c)}+p_{n} T_{n+1}^{(c)}+q_{n} T_{n+1}^{(d)}+q_{n} T_{n}^{(d)}\right) \lambda^{2}+\left(q_{n}-T_{n}^{(c)}\right) \lambda, \\
f_{22}(\lambda, n)= & \left(T_{n}^{(a)} T_{n+1}^{(c)}-q_{n} T_{n}^{(b)} T_{n+1}^{(d)}-T_{n}^{(a)} T_{n}^{(b)} T_{n+1}^{(c)}+T_{n}^{(a)} T_{n+1}^{(d)}-T_{n}^{(a)} T_{n}^{(b)} T_{n+1}^{(d)}-p_{n} T_{n}^{(b)} T_{n+1}^{(c)}\right) \lambda^{2} \\
& +\left(T_{n}^{(a)}-T_{n}^{(a)} T_{n}^{(b)}+T_{n+1}^{(c)}-T_{n}^{(b)} T_{n+1}^{(c)}+T_{n+1}^{(d)}-T_{n}^{(b)} T_{n+1}^{(d)}-q_{n} T_{n}^{(b)}\right) \lambda+\left(1-T_{n}^{(b)}\right) .
\end{aligned}
$$

Then, we find that $f_{11}(\lambda, n)$ and $f_{21}(\lambda, n)$ are two 3thorder polynomial in $\lambda$ and $f_{12}(\lambda, n)$ and $f_{22}(\lambda, n)$ are two 2 th-order polynomial in $\lambda$. By a tedious but direct computation or by a mathematical software, we get that $\lambda_{1}, \lambda_{2}$ are two roots of $f_{i j}(\lambda, n)(1 \leq i, j \leq 2)$. Thus, we may assume

$$
T_{n+1} U_{n} T_{n}^{*}=\operatorname{Det}\left[T_{n}\right] \alpha_{n}
$$

with

$$
\alpha_{n}=\left(\begin{array}{cc}
\alpha_{11}^{(1)} \lambda+\alpha_{11}^{(0)} & \alpha_{12}^{(0)} \\
\alpha_{21}^{(1)} \lambda+\alpha_{21}^{(0)} & \alpha_{22}^{(0)}
\end{array}\right),
$$

where $\alpha_{i 1}^{(1)}, \alpha_{i j}^{(0)}(1 \leq i, j \leq 2)$ are all independent of $\lambda$. Thus, we obtain

$$
T_{n+1} U_{n}=\alpha_{n} T_{n}
$$


By comparing the coefficients of $\lambda^{i}(i=0,1)$, in both sides of equation (97), we get that

$$
\begin{aligned}
& \alpha_{11}^{(1)}=-\frac{T_{n}^{(d)} p_{n}}{p_{n} T_{n}^{(b)}+T_{n}^{(a)} T_{n}^{(b)}-T_{n}^{(a)}}, \\
& \alpha_{11}^{(0)}=0, \\
& \alpha_{12}=1, \\
& \alpha_{21}^{(1)}=\frac{q_{n}-T_{n}^{(c)}}{1-T_{n}^{(b)}}, \\
& \alpha_{21}^{(0)}=0, \alpha_{22}^{(0)}=1 .
\end{aligned}
$$

Hence, we obtain that $\alpha_{n}=\widetilde{U}_{n}$. The proof is finished.

Proposition 6. The matrix $\widetilde{N}_{n}^{(1)}$ given in (80) has the same form as $N_{n}^{(1)}$ in (70) by means of the transformation (89).

Proof. Let us denote

$$
\left(\left(T_{n}\right)_{t}+T_{n} N_{n}^{(1)}\right) T_{n}^{*}=\left(\begin{array}{ll}
g_{11}(\lambda, n) & g_{12}(\lambda, n) \\
g_{21}(\lambda, n) & g_{22}(\lambda, n)
\end{array}\right),
$$

$$
\begin{aligned}
g_{11}(\lambda, n)= & \left(\left(T_{n, t}^{(a)}\left(1-T_{n}^{(b)}\right) T_{n}^{(d)}\right)-T_{n}^{(a)} T_{n, t}^{(b)} T_{n}^{(d)}\left(p_{n-1}-q_{n-1}\right)\right) \lambda^{2}+\left(T_{n, t}^{(a)}\left(1-T_{n}^{(b)}\right)-T_{n}^{(a)} T_{n, t}^{(b)}\right. \\
& +T_{n}^{(a)}\left(1-T_{n}^{(b)}\right) T_{n}^{(c)}-T_{n, t}^{(b)} T_{n}^{(c)}+\frac{1}{2} T_{n}^{(a)}\left(1-T_{n}^{(b)}\right) T_{n}^{(d)}-T_{n, t}^{(b)} T_{n}^{(d)}-T_{n}^{(a)}\left(1-T_{n}^{(b)} p_{n-1}-(1)-T_{n}^{(b)} T_{n}^{(d)} p_{n-1}\right. \\
& \left.+T_{n}^{(a)}\left(1-T_{n}^{(b)}\right) q_{n-1}+\left(1-T_{n}^{(b)} T_{n}^{(d)} q_{n-1}-T_{n}^{(b)} T_{n}^{(c)} q_{n}-T_{n}^{(b)} T_{n}^{(d)} q_{n}\right)\right) \lambda+\frac{1}{2}\left(T_{n}^{(a)}\left(1-T_{n}^{(b)}\right)\right. \\
& \left.-T_{n}^{(b)} T_{n}^{(c)}+T_{n}^{(d)}\left(1-T_{n}^{(b)}\right)\right)+T_{n}^{(c)}-T_{n, t}^{(b)}-\left(1-T_{n}^{(b)}\right) p_{n-1}+\left(1-T_{n}^{(b)}\right) q_{n-1}-T_{n}^{(b)} q_{n}+\frac{1-T_{n}^{(b)}}{2 \lambda}, \\
g_{12}(\lambda, n)= & -\left(T_{n}^{(a)}\right)^{2}+2\left(T_{n}^{(a)}\right)^{2} T_{n}^{(b)}-T_{n, t}^{(a)} T_{n}^{(b)}-\left(T_{n}^{(a)}\right)^{2}\left(T_{n}^{(b)}\right)^{2}+T_{n, t}^{(a)}\left(T_{n}^{(b)}\right)^{2}+T_{n}^{(a)} T_{n, t}^{(b)} \\
& \left.+T_{n}^{(a)} T_{n}^{(b)}\left(p_{n-1}-q_{n-1}\right)-T_{n}^{(a)}\left(T_{n}^{(b)}\right)^{2}\left(p_{n-1}-q_{n-1}\right)+T_{n}^{(a)} T_{n}^{(b)}\left(1-T_{n}^{(b)}\right) q_{n}\right) \lambda+T_{n}^{(a)}\left(3 T_{n}^{(b)}\right. \\
& \left.-2-\left(T_{n}^{(b)}\right)^{2}\right)+T_{n, t}^{(b)}+T_{n}^{(b)}\left(1-T_{n}^{(b)}\right)\left(p_{n-1}-q_{n-1}\right)+T_{n}^{(b)} q_{n}-\frac{1-T_{n}^{(b)}}{\lambda} \\
& \\
g_{21}(\lambda, n)= & T_{n, t}^{(c)} T_{n}^{(d)}-T_{n}^{(c)} T_{n, t}^{(d)}-T_{n}^{(c)} T_{n}^{(d)}\left(p_{n-1}-q_{n-1}\right)-T_{n}^{(c)} T_{n}^{(d)} q_{n}-\left(T_{n}^{(d)}\right)^{2} q_{n} \lambda^{2}+\left(T_{n}^{(c)}\right)^{2}+T_{n, t}^{(c)} \\
& \left.+T_{n}^{(c)} T_{n}^{(d)}-T_{n}^{(c)}\left(p_{n-1}-q_{n-1}\right)-T_{n}^{(c)} q_{n}-2 T_{n}^{(d)} q_{n}\right) \lambda+T_{n}^{(c)}-q_{n} . \\
& \left.\left.+T_{n}^{(d)} q_{n}\right)\right) \lambda+\frac{1}{2}\left(T_{n}^{(a)}\left(T_{n}^{(b)}-1\right)+\left(T_{n}^{(b)}-2\right) T_{n}^{(c)}+T_{n}^{(d)}\left(T_{n}^{(b)}-1\right)+2 q_{n}\right)+\frac{T_{n}^{(b)}-1}{2 \lambda} . \\
& +T_{n}^{(a)} T_{n}^{(c)}\left(T_{n}^{(b)}-1\right)-T_{n}^{(b)} T_{n, t}^{(c)}(1)-T_{n}^{(b)} T_{n, t}^{(d)}+T_{n}^{(b)} T_{n}^{(c)}\left(p_{n-1}-q_{n-1}\right)+T_{n}^{(a)}\left(1-T_{n}^{(b)}\right) q_{n} \\
g_{22}(\lambda, n)= & \left.T_{n}^{(a)} T_{n, t}^{(d)}-T_{n}^{(a)} T_{n}^{(b)} T_{n, t}^{(d)}+T_{n}^{(a)} T_{n}^{(d)}\left(1-T_{n}^{(b)}\right) q_{n}\right) \lambda^{2}+\left(\frac{T_{n}^{(a)} T_{n}^{(d)}\left(T_{n}^{(b)}-1\right)}{2}\right) \\
& \\
& \\
& \\
&
\end{aligned}
$$

Based on (70) and (87), we get

$\left.\sigma_{i, t}(n)=-q_{n}+\left(q_{n}-\frac{1}{\lambda_{i}}+p_{n-1}-q_{n-1}\right) \sigma_{i}[n]+\frac{1}{\lambda_{i}} \sigma_{i}[n]\right)^{2}, \quad i=1,2$.

(101)
Through tediously long calculation, we can find that $g_{i j}\left(\lambda_{i}, n\right)=0(i, j=1,2)$. So, we have

$$
\left(T_{n, t}+T_{n} N_{n}^{(1)}\right) T_{n}^{*}=\operatorname{Det}\left(T_{n}\right) \beta_{n}
$$

where 


$$
\beta_{n}=\left(\begin{array}{cc}
\beta_{11}^{(0)}+\frac{\beta_{11}^{(-1)}}{\lambda} & \frac{\beta_{12}^{(-1)}}{\lambda} \\
\beta_{21}^{(0)} & \beta_{22}^{(0)}+\frac{\beta_{22}^{(-1)}}{\lambda}
\end{array}\right),
$$

and $\beta_{11}^{(0)}, \beta_{11}^{(-1)}, \beta_{12}^{(-1)}, \beta_{21}^{(0)}, \beta_{22}^{(0)}$, and $\beta_{22}^{(-1)}$ are all independent of $\lambda$.

By means of equation (102), we obtain

$$
\left(T_{n, t}+T_{n} N_{n}^{(1)}\right)=\beta_{n} T_{n} .
$$

Comparing the coefficients of $\lambda^{i}(i=-1,0)$ in (104), we have

$$
\begin{aligned}
& \beta_{11}^{(0)}=\tilde{q}_{n-1}-\tilde{p}_{n-1}, \\
& \beta_{11}^{(-1)}=\frac{1}{2}, \\
& \beta_{12}^{(-1)}=-1, \\
& \beta_{21}^{(1)}=-\tilde{q}_{n}, \\
& \beta_{22}^{(0)}=\tilde{q}_{n}, \\
& \beta_{22}^{(-1)}=-\frac{1}{2} .
\end{aligned}
$$

Thus,

$$
\left(T_{n, t}+T \cdot N_{n}^{(1)}\right) T_{n}^{-1}=\widetilde{N}_{n}^{(1)}
$$

The proof is finished.

The transformations (78) and (89), namely, from $\left(\psi_{n} ; p_{n}, q_{n}\right)$ to $\left(\widetilde{\psi}_{n} ; \widetilde{p}_{n}, \widetilde{q}_{n}\right)$ constitute a Darboux-Bäcklund transformation of the NIDDE (69).

In conclusion, according to the Propositions 5 and 6, we have the theorem.

Theorem 2. Every solution $\left(p_{n}, q_{n}\right)^{T}$ of the NLDDE (69) is changed into a new solution $\left(\tilde{p}_{n}, \widetilde{q}_{n}\right)^{T}$ under the Darboux-Bäcklund transformation (89).

\section{Exact Solutions}

Next, we will use the Darboux-Bäcklund transformation (89) to find two solutions of equation (69).

First, we consider a seed solution of (69) (a simple special solution) $\left(p_{n}, q_{n}\right)^{T}=(1,0)^{T}$. Substituting this solution into the corresponding Lax pair (7) and (70), we have

$$
\begin{aligned}
E \psi_{n} & =\left(\begin{array}{ll}
\lambda & 1 \\
0 & 1
\end{array}\right) \psi_{n}, \\
\psi_{n_{t}} & =\left(\begin{array}{cc}
\frac{1}{2 \lambda}-1 & -\frac{1}{\lambda} \\
0 & -\frac{1}{2 \lambda}
\end{array}\right) \psi_{n} .
\end{aligned}
$$

Solving above two equations, we arrive at the solutions

$$
\begin{aligned}
& \psi_{n}=\left(\begin{array}{c}
\psi_{n}^{(1)}(\lambda, t) \\
\psi_{n}^{(2)}(\lambda, t)
\end{array}\right)=\left(\begin{array}{c}
\frac{\lambda^{n} \exp \left(\left(\left(\lambda-2 \lambda^{2}\right) t\right) /\left(2 \lambda^{2}\right)\right)-\exp (-t / 2 \lambda)}{\lambda-1} \\
\exp \left(-\frac{t}{2 \lambda}\right)
\end{array}\right), \\
& \phi_{n}=\left(\begin{array}{c}
\phi_{n}^{(1)}(\lambda, t) \\
\phi_{n}^{(2)}(\lambda, t)
\end{array}\right)=\left(\begin{array}{c}
\frac{-\lambda^{n} \exp \left(\left(\left(\lambda-2 \lambda^{2}\right) t\right) /\left(2 \lambda^{2}\right)\right)-\exp (-t / 2 \lambda)}{\lambda-1} \\
-\exp \left(-\frac{t}{2 \lambda}\right)
\end{array}\right) .
\end{aligned}
$$

Then, we obtain

$$
\sigma_{i}[n]=\frac{\psi_{n}^{(2)}\left(\lambda_{i}, t\right)-\kappa_{i} \phi_{n}^{(2)}\left(\lambda_{i}, t\right)}{\psi_{n}^{(1)}\left(\lambda_{i}, t\right)-\kappa_{i} \phi_{n}^{(1)}\left(\lambda_{i}, t\right)}=\frac{\left(1+\kappa_{i}\right) \lambda_{i}^{n} \exp \left(-t / 2 \lambda_{i}\right)\left(1-\lambda_{i}\right)}{\left(1-\kappa_{i}\right) \lambda_{i}^{n} \exp \left(\left(\lambda_{i}-2 \lambda_{i}^{2}\right) /\left(2 \lambda_{i}^{2}\right)\right)+\left(1+\kappa_{i}\right) \exp \left(-t / 2 \lambda_{i}\right)}, \quad i=1,2 .
$$


Using the Darboux-Bäcklund transformation (89) and with the help of mathematical software "Mathematica," we obtain an exact solution of (69):

$$
\begin{aligned}
\tilde{p}_{n} & =-\frac{T_{n}^{(d)}}{T_{n}^{(b)}+T_{n}^{(a)} T_{n}^{(b)}-T_{n}^{(a)}} \\
& =-\frac{\left(\lambda_{1} \sigma_{2}[n]-\lambda_{2} \sigma_{1}[n]\right)^{2}\left(\lambda_{1}-\lambda_{2}-\left(\lambda_{1} \sigma_{2}[n]-\lambda_{2} \sigma_{1}[n]\right)\right)}{\lambda_{1} \lambda_{2}\left(\sigma_{1}[n]-\sigma_{2}[n]\right)\left(\lambda_{1}-\lambda_{2}\right)\left(\lambda_{1} \sigma_{2}[n]-\lambda_{2} \sigma_{1}[n]\right)+\left(\sigma_{1}[n]-\sigma_{2}[n]\right)\left(\left(\lambda_{1}-\lambda_{2}-1\right)\right)}, \\
\tilde{q}_{n} & =\frac{T_{n}^{(c)}}{1-T_{n}^{(b)}}=\frac{\left(\lambda_{1}-\lambda_{2}-\left(\lambda_{1} \sigma_{2}[n]-\lambda_{2} \sigma_{1}[n]\right)\left(\lambda_{1}-\lambda_{2}\right)\right.}{\left(\sigma_{1}[n]-\sigma_{2}[n]\right)\left(\lambda_{2} \sigma_{1}[n]-\lambda_{1} \sigma_{2}[n]\right) \lambda_{1} \lambda_{2}\left(\sigma_{1}\right)[n]-\sigma_{2}[n]} .
\end{aligned}
$$

Then, it easy to verify that $\left(p_{n}, q_{n}\right)^{T}=(1,-1)^{T}$ is another Set seed solution of (69). Substituting it into the corresponding Lax pair, it is found that

$$
\begin{aligned}
E \psi_{n} & =\left(\begin{array}{cc}
1 & 1 \\
-\lambda & 1
\end{array}\right) \psi_{n}, \\
\psi_{n_{t}} & =\left(\begin{array}{cc}
\frac{1}{2 \lambda}-2 & -\frac{1}{\lambda} \\
1 & -\frac{1}{2 \lambda}
\end{array}\right) \psi_{n} .
\end{aligned}
$$

$$
\begin{aligned}
& \chi_{1}(\lambda, t)=\exp \left(\frac{t\left(-3 \lambda-\sqrt{\lambda^{2}-6 \lambda+1}\right.}{2 \lambda}\right), \\
& \chi_{2}(\lambda, t)=\exp \left(\frac{t(-) 3 \lambda+\sqrt{\lambda^{2}-6 \lambda+1}}{2 \lambda}\right),
\end{aligned}
$$

$h_{1}(\lambda, n)=\frac{2^{-n-1}\left(1-\lambda+\sqrt{\lambda^{2}-6 \lambda+1}\right)\left(1+\lambda-\sqrt{\lambda^{2}-6 \lambda+1}\right)^{n}}{\sqrt{\lambda^{2}-6 \lambda+1}}+\frac{2^{-n-1}\left(-1+\lambda+\sqrt{\lambda^{2}-6 \lambda+1}\right)\left(1+\lambda+\sqrt{\lambda^{2}-6 \lambda+1}\right)^{n}}{\sqrt{\lambda^{2}-6 \lambda+1}}$,

$$
h_{2}(\lambda, n)=\frac{2^{-n} \lambda}{\sqrt{\lambda^{2}-6 \lambda+1}}\left(\left(1+\lambda-\sqrt{\lambda^{2}-6 \lambda+1}\right)^{n}-\left(1+\lambda+\sqrt{\lambda^{2}-6 \lambda+1}\right)^{n}\right) \text {, }
$$$$
l_{1}(\lambda, n)=\frac{2^{n}}{\sqrt{\lambda^{2}-6 \lambda+1}}\left(\left(1+\lambda-\sqrt{\lambda^{2}-6 \lambda+1}\right)^{n}-\left(1+\lambda+\sqrt{\lambda^{2}-6 \lambda+1}\right)^{n}\right),
$$$$
l_{2}(\lambda, n)=\frac{2^{-n-1}\left(-1+\lambda+\sqrt{\lambda^{2}-6 \lambda+1}\right)\left(1+\lambda-\sqrt{\lambda^{2}-6 \lambda+1}\right)^{n}}{\sqrt{\lambda^{2}-6 \lambda+1}}+\frac{2^{-n-1}\left(1-\lambda+\sqrt{\lambda^{2}-6 \lambda+1}\right)\left(1+\lambda+\sqrt{\lambda^{2}-6 \lambda+1}\right)^{n}}{\sqrt{\lambda^{2}-6 \lambda+1}} .
$$ 
By means of mathematical software "Mathematica," we

where may get that two real linear independent solutions as follows:

$$
\begin{aligned}
& \widetilde{\psi}_{n}=\left(\begin{array}{c}
\widetilde{\psi}_{n}^{(1)}(\lambda, t) \\
\left.\widetilde{\psi}_{n}^{(2)}(\lambda, t)\right)
\end{array}\right), \\
& \widetilde{\phi}_{n}=\left(\begin{array}{c}
\widetilde{\phi}_{n}^{(1)}(\lambda, t) \\
\widetilde{\phi}_{n}^{(2)}(\lambda, t)
\end{array}\right),
\end{aligned}
$$

$$
\begin{aligned}
& \widetilde{\psi}_{n}^{(1)}(\lambda, t)=h_{1}(\lambda, n)\left(\frac{(\lambda-1) \chi_{1}(\lambda, n)+(\lambda+1) \chi_{2}(\lambda, n)}{2 \sqrt{\lambda^{2}-6 \lambda+1}}+\frac{\chi_{1}(\lambda, n)+\chi_{2}(\lambda, n)}{2}\right)+h_{2}(\lambda, n)\left(\frac{\chi_{1}(\lambda, n)-\chi_{2}(\lambda, n)}{2 \sqrt{\lambda^{2}-6 \lambda+1}}\right) \\
& \widetilde{\psi}_{n}^{(2)}(\lambda, t)=h_{1}(\lambda, n)\left(\frac{\lambda \chi_{1}(\lambda, n)-\lambda \chi_{2}(\lambda, n)}{2 \sqrt{\lambda^{2}-6 \lambda+1}}\right)+h_{2}(\lambda, n)\left(\frac{(1-\lambda) \chi_{1}(\lambda, n)-(\lambda-1) \chi_{2}(\lambda, n)}{2 \sqrt{\lambda^{2}-6 \lambda+1}}+\frac{\chi_{1}(\lambda, n)+\chi_{2}(\lambda, n)}{2}\right) \\
& \tilde{\phi}_{n}^{(1)}(\lambda, t)=l_{1}(\lambda, n)\left(\frac{(\lambda-1) \chi_{1}(\lambda, n)+(\lambda+1) \chi_{2}(\lambda, n)}{2 \sqrt{\lambda^{2}-6 \lambda+1}}+\frac{\chi_{1}(\lambda, n)+\chi_{2}(\lambda, n)}{2}\right)+l_{2}(\lambda, n)\left(\frac{\chi_{1}(\lambda, n)-\chi_{2}(\lambda, n)}{2 \sqrt{\lambda^{2}-6 \lambda+1}}\right) \\
& \tilde{\phi}_{n}^{(2)}(\lambda, t)=l_{1}(\lambda, n)\left(\frac{\lambda \chi_{1}(\lambda, n)-\lambda \chi_{2}(\lambda, n)}{2 \sqrt{\lambda^{2}-6 \lambda+1}}\right)+l_{2}(\lambda, n)\left(\frac{(1-\lambda) \chi_{1}(\lambda, n)-(\lambda-1) \chi_{2}(\lambda, n)}{2 \sqrt{\lambda^{2}-6 \lambda+1}}+\frac{\chi_{1}(\lambda, n)+\chi_{2}(\lambda, n)}{2}\right) .
\end{aligned}
$$

Based on (87), we have

$$
\tilde{\sigma}_{i}[n]=\frac{\widetilde{\psi}_{n}^{(2)}\left(\lambda_{i}, t\right)-\gamma_{i} \widetilde{\phi}_{n}^{(2)}\left(\lambda_{i}, t\right)}{\widetilde{\psi}_{n}^{(1)}\left(\lambda_{i}, t\right)-\gamma_{i} \widetilde{\phi}_{n}^{(1)}\left(\lambda_{i}, t\right)}, \quad i=1,2 .
$$

Though the Darboux-Bäcklund transformation (89), we arrive at another exact solution of (69):

$$
\begin{aligned}
& \tilde{p}_{n}=-\frac{T_{n}^{(d)}}{T_{n}^{(b)}+T_{n}^{(a)} T_{n}^{(b)}-T_{n}^{(a)}}=\frac{\left(\lambda_{1} \widetilde{\sigma}_{2}[n]-\lambda_{2} \widetilde{\sigma}_{1}[n]\right)\left(-\lambda_{1}+\lambda_{2}+\lambda_{1} \widetilde{\sigma}_{2}[n]-\lambda_{2} \widetilde{\sigma}_{1}[n]\right)}{\lambda_{1} \lambda_{2}\left(\widetilde{\sigma}_{1}[n]-\widetilde{\sigma}_{2}[n]\right)\left(\lambda_{1}-\lambda_{2}+\widetilde{\sigma}_{1}[n]-\widetilde{\sigma}_{2}[n]\right)}, \\
& \widetilde{q}_{n}=-\frac{1+T_{n}^{(c)}}{1-T_{n}^{(b)}}=\frac{\left(\lambda_{1}-\lambda_{2}+\lambda_{2} \widetilde{\sigma}_{1}[n]-\lambda_{1} \widetilde{\sigma}_{2}[n]\right)\left(\lambda_{1} \lambda_{2}\left(\widetilde{\sigma}_{1}[n]-\widetilde{\sigma}_{2}[n]\right)-\left(\lambda_{1}-\lambda_{2}\right) \widetilde{\sigma}_{1}[n] \widetilde{\sigma}_{2}[n]\right)}{\lambda_{1} \lambda_{2}\left(\widetilde{\sigma}_{1}[n]-\widetilde{\sigma}_{2}[n]\right)} .
\end{aligned}
$$

\section{Conclusions and Remarks}

In this paper, we have deduced two hierarchies of NIDDEs from a discrete matrix spectral problem by the discrete zero curvature equation. The obtained hierarchies, respectively, work in concert with positive power and negative power expansions of Lax operators with respect to the spectral parameter. Two bi-Hamiltonian forms for the obtained integrable hierarchies are given by the discrete trace identity. And then, the Liouville integrability of the obtained hierarchies is demonstrated. Furthermore, by the aid of a gauge transformation of the Lax pair, a Darboux-Bäcklund transformation for the first NIDDE in the negative integrable hierarchy was presented. Applying the obtained Darboux-Bäcklund transformation and "Mathematica," we get two exact solutions. These solutions also are called one-fold solutions. This Darboux-Bäcklund transformation is continuously done $N$ times, then $N$-fold solution of (69) can be derived. Besides, we can get the Darboux-Bäcklund transformation of the first NIDDE (27) in the positive hierarchy in a similar way. Recently, in the soliton theory, some new types of explicit solutions for the continuous soliton equations have been found, for instance, abundant lump solutions and interaction solutions [23-26]. For the NIDDE (69), explicit solutions of these types can also be researched. These results will appear in later papers.

In addition, many interesting problems deserve further investigation for the NIDDEs in the obtained hierarchies (25) and (68), such as symmetries constraint, integrable coupling systems by semidirect sums of Lie algebra, symmetries, and master symmetries. 


\section{Data Availability}

The data used to support the findings of this study are available from the corresponding author on reasonable request.

\section{Conflicts of Interest}

The authors declare that there are no conflicts of interest regarding the publication of this paper.

\section{Acknowledgments}

This work was supported by the Youth Science Foundation Project of China (Grant no. 11805114).

\section{References}

[1] M. J. Ablowitz and P. A. Clarkson, Solitons, Nonlinear Evolution Equations and Inverse Scattering, Cambridge University Press, Cambridge, England, 1991.

[2] M. Toda, Theory of Nonlinear Lattice, Springer-Verlag, Berlin, Germany, 2 edition, 1989.

[3] H. Flaschka, "On the Toda lattice. II: inverse-scattering solution," Progress of Theoretical Physics, vol. 51, no. 3, pp. 703-716, 1974.

[4] W.-X. Ma and X.-X. Xu, "A modified Toda spectral problem and its hierarchy of bi-Hamiltonian lattice equations," Journal of Physics A: Mathematical and General, vol. 37, no. 4, pp. 1323-1336, 2004.

[5] X.-X. Xu, H.-X. Yang, and Y.-P. Sun, "Darboux transformation of the modified Toda lattice equation," Modern Physics Letters B, vol. 20, no. 11, pp. 641-648, 2006.

[6] I. Merola, O. Ragnisco, and T. Gui-Zhang, "A novel hierarchy of integrable lattices," Inverse Problems, vol. 10, no. 6, pp. 1315-1334, 1994.

[7] X.-X. Xu, "A deformed reduced semi-discrete Kaup-Newell equation, the related integrable family and Darboux transformation," Applied Mathematics and Computation, vol. 251, pp. 275-283, 2015.

[8] X. Geng, H. H. Dai, and C. Cao, "Algebro-geometric constructions of the discrete Ablowitz-Ladik flows and applications," Journal of Mathematical Physics, vol. 44, no. 10, pp. 4573-4588, 2003.

[9] T. Tsuchida, "Integrable discretizations of derivative nonlinear Schr dinger equations," Journal of Physics A: Mathematical and General, vol. 35, no. 36, pp. 7827-7847, 2002.

[10] A. Pickering and Z.-N. Zhu, "New integrable lattice hierarchies," Physics Letters A, vol. 349, no. 6, pp. 439-445, 2006.

[11] G. Z. Tu, "A trace identity and its applications to theory of discrete integrable systems," Journal of Physics A: Mathematical and General, vol. 23, pp. 3903-3922, 1990.

[12] D. Zhang and D. Chen, "Hamiltonian structure of discrete soliton systems," Journal of Physics A: Mathematical and General, vol. 35, no. 33, pp. 7225-7241, 2002.

[13] W.-X. Ma and X.-X. Xu, "Positive and negative hierarchies of integrable lattice models associated with a Hamiltonian pair," International Journal of Theoretical Physics, vol. 43, no. 1, pp. 219-235, 2004.

[14] M. Blaszak and K. Marciniak, "R-matrix approach to lattice integrable systems," Journal of Mathematical Physics, vol. 35, pp. 4661-4682, 1994.
[15] D. Y. Chen, "Backlund transformation and Nsoliton solutions," Journal for Research in Mathematics Education, vol. 25, pp. 479-488, 2005.

[16] H. D. Wahlquist and F. B. Estabrook, "Bäcklund transformation for solutions of the korteweg-de Vries equation," Physical Review Letters, vol. 31, no. 23, pp. 1386-1390, 1973.

[17] V. B. Matveev and M. A. Salle, Darboux Transformations and Solitons, Springer, Berlin,Gemany, 1991.

[18] R. Hirota, "A new form of backlund transformations and its relation to the inverse scattering problem," Progress of Theoretical Physics, vol. 52, no. 5, pp. 1498-1512, 1974.

[19] C. H. Gu, H. S. Hu, and Z. X. Zhou, Soliton Theory and its Application, Zhejiang Science and Technology Publishing House, Berlin,Germany, 1995.

[20] Y. T. Wu and X. G. Geng, "A new hierarchy of integrable differential-difference equations and Darboux transformation," Journal of Physics A: Mathematical and General, vol. 31, pp. 677-684, 1998.

[21] X.-X. Xu and Y.-P. Sun, "An integrable coupling hierarchy of Dirac integrable hierarchy, its Liouville integrability and Darboux transformation," The Journal of Nonlinear Sciences and Applications, vol. 10, no. 06, pp. 3328-3343, 2017.

[22] R.-W. Lu, X.-X. Xu, and N. Zhang, "Construction of solutions for an integrable differential-difference equation by DarbouxBäcklund transformation," Applied Mathematics and Computation, vol. 361, pp. 389-397, 2019.

[23] W. X. Ma, "Abundant lumps and their interaction solutions of (3+1)-dimensional linear PDEs," Journal of Geometry and Physics, vol. 133, pp. 45-54, 2018.

[24] S.-T. Chen and W.-X. Ma, "Lump solutions to a generalized Bogoyavlensky-Konopelchenko equation," Frontiers of Mathematics in China, vol. 13, no. 3, pp. 525-534, 2018.

[25] W. X. Ma, J. Li, and C. M. Khalique, "A study on lump solutions to a generalized Hirota-Satsuma-Ito equation in (2+1)-dimensions," Complexity, vol. 2018, Article ID 9059858, 7 pages, 2018.

[26] J.-Y. Yang, W.-X. Ma, and Z. Qin, "Lump and lump-soliton solutions to the $\$ \$(2+1) \$ \$(2+1)$-dimensional Ito equation," Analysis and Mathematical Physics, vol. 8, no. 3, pp. 427-436, 2018. 